\title{
Jahn-Teller Splitting in Single Adsorbed Molecules Revealed by Isospin-Flip Excitations
}

\author{
Jens Kügel, ${ }^{1, *}$ Pin-Jui Hsu, ${ }^{1, \dagger}$ Markus Böhme, ${ }^{1}$ Kathrin Schneider, ${ }^{1, \$}$ Jacob Senkpiel, ${ }^{1, \S}$ David Serrate, ${ }^{2,3, \|}$ \\ Matthias Bode, ${ }^{1,4}$ and Nicolás Lorente ${ }^{5,6}$ \\ ${ }^{1}$ Physikalisches Institut, Experimentelle Physik II, Universität Würzburg, Am Hubland, 97074 Würzburg, Germany \\ ${ }^{2}$ Instituto de Nanociencia de Aragón, Laboratorio de Microscopias Avanzadas, University of Zaragoza, \\ E-50018 Zaragoza, Spain \\ ${ }^{3}$ Departamento Física Materia Condensada, University of Zaragoza, E-50018 Zaragoza, Spain \\ ${ }^{4}$ Wilhelm Conrad Röntgen-Center for Complex Material Systems (RCCM), Universität Würzburg, Am Hubland, \\ D-97074 Würzburg, Germany \\ ${ }^{5}$ Centro de Física de Materiales CFM/MPC (CSIC-UPV/EHU), 20018 Donostia-San Sebastián, Spain \\ ${ }^{6}$ Donostia International Physics Center (DIPC), 20018 Donostia-San Sebastián, Spain
}

(Received 15 May 2018; revised manuscript received 11 September 2018; published 29 November 2018)

Scanning tunneling spectroscopy measurements of $\mathrm{Mn}$ phthalocyanine $(\mathrm{MnPc})$ molecules adsorbed on $(\sqrt{3} \times \sqrt{3})$ surface alloys show single inelastic steps at exclusively positive or negative bias strongly depending on the tip position. This is in contrast to conventional molecular excitation thresholds, which are independent of the current direction and therefore always occur at both positive and negative bias. This polarity selectivity is found to coincide with the spatial distribution of occupied and empty orbitals. Because of the interaction with the substrate, charge transfer into the doubly degenerate $d_{\pi}$ orbitals of MnPc takes place. The resulting Jahn-Teller effect lifts the degeneracy and leads to an isospin- or pseudospin-flip excitation, the inelastic analogue of an orbital Kondo resonance.

DOI: 10.1103/PhysRevLett.121.226402

In the past decades, the spin Kondo effect was the subject of numerous studies [1-3]. Its hallmark is a resonance at the Fermi level $E_{F}$ (see green curve in Fig. 1) which emerges as itinerant electrons of the host material screen uncompensated magnetic moments in a spin-degenerate localized orbital. The energy gain of the resulting many-body Kondo state is typically expressed in terms of a Kondo temperature $T_{K}$. As schematically represented in blue in Fig. 1, the application of a magnetic field lifts spin degeneracy and leads to a splitting of the Kondo resonance into fully spinpolarized peaks [4]. If the Zeeman energy approaches (red curve) or even exceeds (black curve) $k_{B} T_{K}$, where $k_{B}$ is the Boltzmann constant, the resonance is strongly reduced and eventually disappears. In the latter case, the only detectable feature in a spectroscopic response will be related to inelastic spin-flip excitations of the localized orbital [4].

The spin is not the only degree of freedom which can lead to a Kondo effect. For example, the presence of degenerate states with different orbital quantum number can also give rise to the so-called orbital Kondo effect [5-8]. Similar to the spin Kondo effect, the orbital Kondo effect manifests itself in a sharp resonance at $E_{F}$, which splits as orbital degeneracy is lifted, for example, due to the Jahn-Teller effect, which is common in phthalocyanine molecules $[9,10]$. Eventually the orbital Kondo effect is quenched if the Jahn-Teller energy leads to a level splitting much larger than $k_{B} T_{K}$. In analogy to the abovementioned spin-flip excitation, we may expect that hopping between the orbitals can be excited by inelastic electron scattering processes, for example, in a scanning tunneling spectroscopy (STS) experiment. Since such a scattering process would only change the orbital degree of freedom but leave the spin unchanged, it has been coined "isospin flip" (or pseudospin flip) [11]. In contrast to spin-flip excitations, however, very little is known about these so-called isospin-flip excitations.

In this study, we report on the observation of an unusual spatial variation of the inelastic (IE STS) signal observed on single MnPc molecules adsorbed on $(\sqrt{3} \times \sqrt{3})$ surface alloys of heavy post-transition metals with noble metal fcc(111) surfaces. These surfaces have unit cell sizes comparable with the dimensions of Pc molecules, thereby breaking the symmetry of the molecules. On these distorted molecules we measure tunneling spectra which are characterized by abrupt rises of the conductance at either

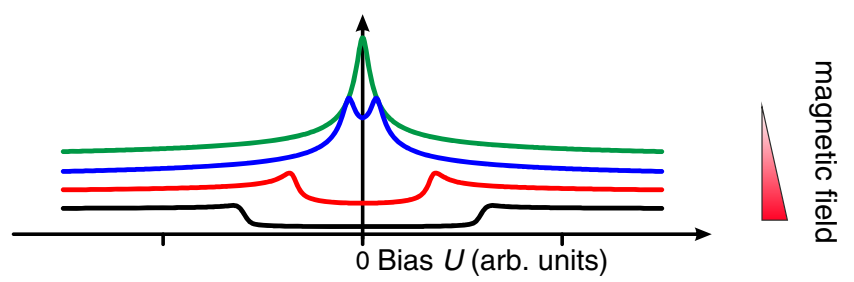

FIG. 1. Schematic representation of the magnetic field dependence of the spin Kondo effect. The spectra are displaced for visualization purpose. The lowest spectra correspond to the largest magnetic field. 
positive or negative bias polarity depending on the tip position. However, the appearance of inelastic steps at both polarities is customarily used as a sign of IE STS [12-21]. This spatial dependence of the IE STS points at an orbital origin of the inelastic process. Indeed, $\mathrm{MnPc}$ shows a doubly degenerate electronic structure described by the half filling of its $d_{\pi}$ orbitals. Our theoretical analysis shows that the threshold bias matches the energy difference of the orbitals, which in the absence of degeneracy lifting would lead to an orbital Kondo effect due to the charge fluctuation between orbitals caused by substrate electrons [5-8]. The large charging energy of the MnPc $d_{\pi}$ orbitals leads to the exclusive filling of one or the other orbital. The dynamical orbital filling behaves like a spin- $1 / 2$ system and can be defined as an isospin [22]. The resulting isospin-flip excitation energy corresponds to the lifting of orbital degeneracy caused by the Jahn-Teller energy of the adsorbed MnPc.

Experiments were performed in two low-temperature STMs 1 and 2 operating at $T_{1} \approx 5 \mathrm{~K}$ and $T_{2} \approx 1.2 \mathrm{~K}$. Dilute amounts of $\mathrm{MnPc}$ were dosed at ambient temperature onto $\mathrm{BiCu}_{2}, \mathrm{BiAg}_{2}$, or $\mathrm{SbAg}_{2}$, prepared by depositing $\mathrm{Bi}$ or $\mathrm{Sb}$ on either $\mathrm{Cu}(111)$ or $\mathrm{Ag}(111)$ [23,24]. STS data were acquired by adding a small modulation to the bias voltage $U$ (frequency $\nu_{1}=811.7 \mathrm{~Hz}$ and $\nu_{2}=893 \mathrm{~Hz}$ ), such that differential conductance $d I / d U$ spectra as well as $d I / d U$ maps can be detected by lock-in techniques.

Figure 2(a) shows a topographic image of a $\mathrm{MnPc}$ molecule on the atomically resolved $(\sqrt{3} \times \sqrt{3})$ reconstructed $\mathrm{BiCu}_{2} / \mathrm{Cu}(111)$ surface alloy $[25,26]$. The molecule center as defined by the molecular arms is positioned on top of a $\mathrm{Bi}$ atom (see white dashed lines) with the arms pointing along high-symmetry directions of the substrate. To analyze the electronic features of $\mathrm{MnPc}$, Fig. 2(b) displays the topography together with $d I / d U$ maps taken at Fig. 2(c) $U=-0.45 \mathrm{~V}$, Fig. 2(d) $U=$ $-13 \mathrm{mV}$, Fig. 2(e) $U=+13 \mathrm{mV}$, and Fig. 2(f) $U=$ $+0.30 \mathrm{~V}$. All data are arranged such that the molecule orientation and the crystallographic axes of the substrate in Figs. 2(b)-2(f) point in the same directions. All $d I / d U$ maps presented in Figs. 2(c)-2(f) feature six lobes, irrespective of the specific bias voltage. However, the spatial distribution of these lobes with respect to the underlying $\mathrm{BiCu}_{2}$ surface alloy is quite different. The occupied LDOS exhibits maxima along $\langle 10 \overline{1}\rangle$ directions but is minimal along $\langle\overline{1} 2 \overline{1}\rangle$ directions [cf. Figs. 2(c) and 2(d)], and vice versa for the unoccupied energy range [cf. Figs. 2(e) and 2(f)].

This appearance of the MnPc molecule was further investigated by tunneling spectra taken at the positions marked by red and blue crosses in Figs. 2(c) and 2(e), where we find local minima and maxima of the LDOS in the occupied [Figs. 2(c) and 2(d)] and empty states [Figs. 2(e) and 2(f)]. Indeed, the overview spectra plotted in Fig. 2(g) reveal striking differences. Whereas a maximum at $U \approx-0.43 \mathrm{~V}$ together with a small resonance slightly below the Fermi energy is observed at the position

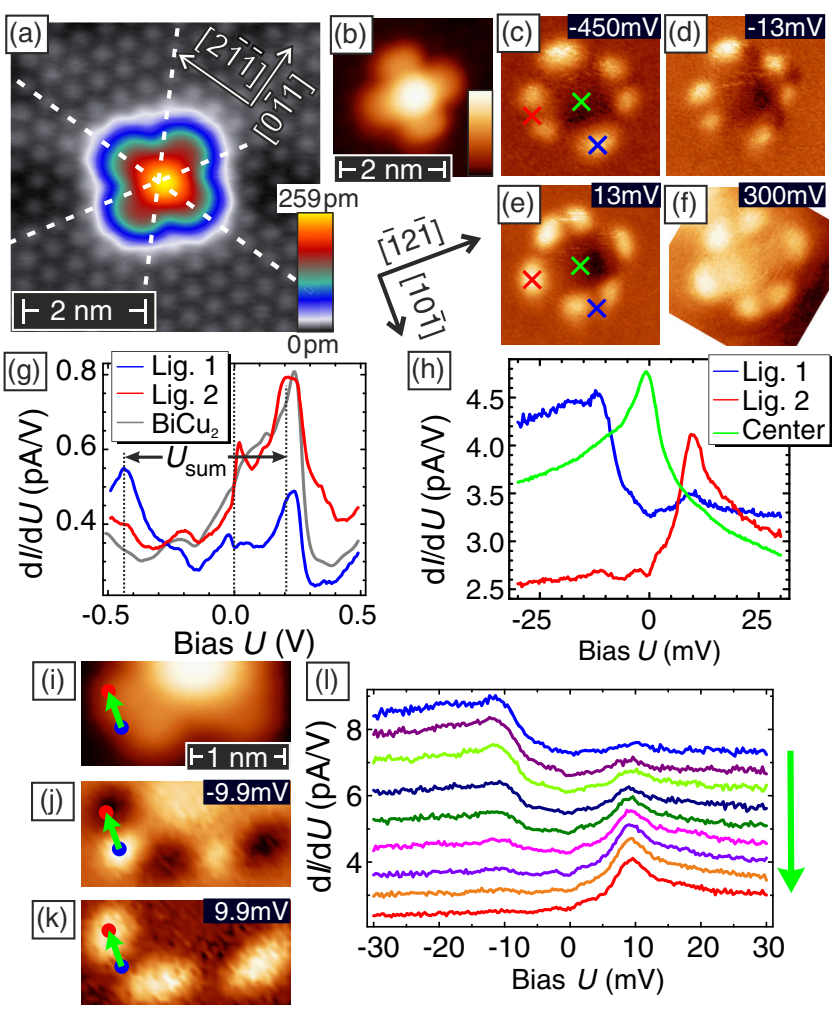

FIG. 2. (a) Topographic image of MnPc on the atomically resolved $\mathrm{BiCu}_{2}$ alloy (scan parameters are $U=-0.2 \mathrm{~V}$, $I=1.0 \mathrm{nA}$ ). (b) Topography of the MnPc molecule together with $d I / d U$ maps taken at (c) $U=-0.45 \mathrm{~V}$, (d) $U=-13 \mathrm{mV}$, (e) $U=+13 \mathrm{mV}$, and (f) $U=+0.30 \mathrm{~V}$. (g) Spectroscopy curves taken at the position marked by crosses in the corresponding color in (c) and (e) together with a spectrum of the substrate (gray) (parameters are $U=-0.5 \mathrm{~V}, I=0.2 \mathrm{nA}, U_{\bmod }=$ $10 \mathrm{mV}, T=5 \mathrm{~K}$ ). (h) Spectroscopy curves of the two ligand states (red and blue) and the central metal ion (green) ( $\left.U=30 \mathrm{mV}, \quad I=100 \mathrm{pA}, \quad U_{\bmod }=1 \mathrm{mV}, \quad T=1.2 \mathrm{~K}\right)$. (i) Topography together with $d I / d U$ maps taken at (j) $U=$ $-9.9 \mathrm{mV}$ and $(\mathrm{k}) U=9.9 \mathrm{mV}$, which was extracted from a full spectroscopy measurement $\left(U=30 \mathrm{mV}, I=100 \mathrm{pA}, U_{\bmod }=\right.$ $1 \mathrm{mV}, T=1.2 \mathrm{~K}$ ). (l) Spectroscopy curves extracted from the full spectroscopy in a line between the red and black point shown in (i)-(k) (distance increment between consecutive curves is $50 \mathrm{pm}$ ).

of the blue cross, the red curve exhibits a pronounced maximum in the unoccupied states at $U \approx 0.2 \mathrm{~V}$ and a resonance slightly above $E_{F}$. We would like to note that both spectra show a van Hove singularity at $U=0.23 \mathrm{~V}$, which originates from the band edge of the Rashba-split $\mathrm{BiCu}_{2} / \mathrm{Cu}(111)$ surface state $[25,26]$ [cf. gray spectrum in Fig. 2(g)]. This substrate-related electronic feature is particularly well visible in $d I / d U$ spectra taken at the blue cross where no molecule-related states are detected. In contrast, in the red spectrum the superposition of the unoccupied molecular state with the energetically close-by Rashba surface state leads to a broadened peak.

The two features close to $E_{F}$ are presented at higher energy resolution in Fig. 2(h). In addition, we plot a $d I / d U$ 
spectrum taken at the central metal ion of the $\mathrm{MnPc}$ (green curve), which shows a resonance at the Fermi energy, an indicator for a Kondo screening of the $d$ orbital $[27,28]$. Interestingly, the $d I / d U$ spectra measured at the different positions on the ligand of the molecule (red and blue curves) both show a steplike behavior separated by roughly $|\Delta U| \approx$ $9 \mathrm{meV}$ from the Fermi energy. In contrast to IE STS [15], however, where the two steps are positioned symmetrically around $E_{F}$, the ligand spectra in Fig. 2(h) exhibit a peak in either the occupied or unoccupied energy range.

To further analyze this anomaly, we took a grid of $40 \times 20$ spectroscopy curves. The topography and $d I / d U$ maps measured at $U= \pm 9.9 \mathrm{mV}$ of the bottom half of a molecule are presented in Figs. 2(i)-2(k), respectively. A sequence of spectroscopy curves along the line between the blue and red point are plotted in Fig. 2(1). The spectrum taken at the blue point exhibits a steplike feature below $E_{F}$. As the STM tip is moved towards the red point, this feature continuously decreases. Simultaneously, the intensity of the steplike feature above $E_{F}$ increases until it completely dominates the red spectrum.

The data presented so far reveal that $\mathrm{MnPc}$ on $\mathrm{BiCu}_{2}$ shows two orbital states, an occupied and an unoccupied one, which can be observed at different locations of its ligand. We find that each of these orbital states appears to be related to a steplike feature close to $E_{F}$. To test if these features are influenced or even caused by the strong spinorbit coupling present in the $\mathrm{BiCu}_{2} / \mathrm{Cu}(111)$ surface alloy, we also investigated the electronic structure of $\mathrm{MnPc}$ on isostructural surfaces with a much higher and much lower Rashba energy, i.e., $\mathrm{BiAg}_{2}$ and $\mathrm{SbAg}_{2}$, respectively.

The data measured on $\mathrm{BiAg}_{2}$, which show a very similar behavior as the results on $\mathrm{BiCu}_{2}$ presented in Fig. 2, can be found in the Supplemental Material [29]. Figure 3(a) shows a topographic image of $\mathrm{MnPc}$ on $\mathrm{SbAg}_{2}$ which-in contrast to the other two surface alloys-is not subject to large spin-orbital coupling or Rashba effects [41]. Similar to $\mathrm{BiCu}_{2}$, the center of the $\mathrm{MnPc}$ molecule is above an antimony atom, but the arms of the molecule are rotated by $45^{\circ}$ with respect to the substrate's highsymmetry directions. In spite of this slightly different adsorption geometry, the combination of topographic data [Fig. 3(b)], $d I / d U$ maps taken at bias voltages of $U=$ $-20 \mathrm{mV}$ and $U=+20 \mathrm{mV}$ [Figs. 3(c) and 3(d)], and tunneling spectra [Fig. 3(e)] presents a picture which is strikingly similar to the observations made for $\mathrm{MnPc}$ on $\mathrm{BiCu}_{2}$ and $\mathrm{BiAg}_{2}$. In any case, we observe steplike features in $d I / d U$ spectra measured on the MnPc ligand. These features appear symmetrically around and close to the Fermi energy (typically at $U \approx \pm 10 \mathrm{mV}$ ) and exhibit a characteristic spatial dependence where the intensity of the occupied and the unoccupied feature alternate. The similarity of results obtained on $\mathrm{SbAg}_{2}$ on one side and on $\mathrm{BiCu}_{2}$ and $\mathrm{BiAg}_{2}$ on the other side permits us to disregard spin-orbit effects as a potential origin of the spatially dependent stepped features.

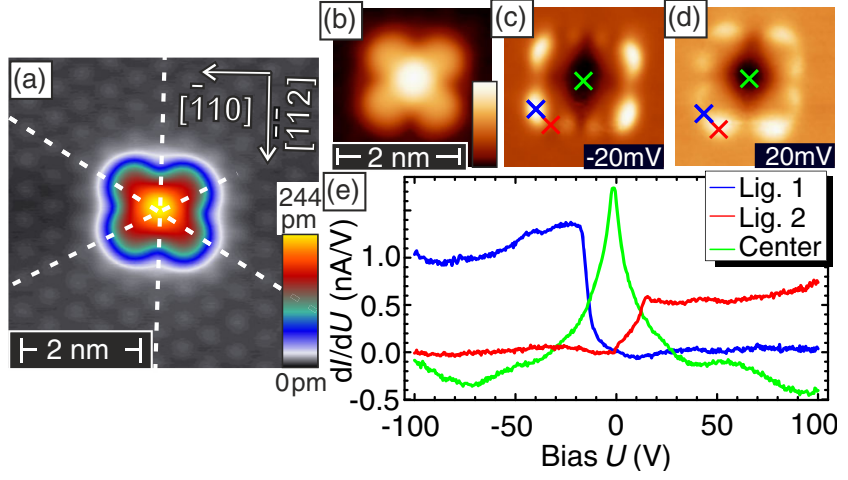

FIG. 3. (a) Topographic image of MnPc on the atomically resolved $\mathrm{SbAg}_{2}$ alloy (scan parameters are $U=0.1 \mathrm{~V}, I=$ $0.1 \mathrm{nA}$ ). (b) Topography of the MnPc molecule together with $d I / d U$ maps taken at (c) $U=-20 \mathrm{mV}$ and (d) $U=20 \mathrm{mV}$. (e) Spectroscopy curves taken at the position marked by crosses in the corresponding color in (c) and (d) $[U=+0.1 \mathrm{~V}$ (blue and green curve), and $U=-0.1 \mathrm{~V}$ (red curve), $I=0.2 \mathrm{nA}$, $\left.U_{\text {mod }}=1 \mathrm{mV}, T=5 \mathrm{~K}\right]$.

The experimental data can be rationalized by studying the molecular orbitals close to $E_{F}$. Figure 4(a) shows the adsorption geometry of $\mathrm{MnPc}$ on $\mathrm{SbAg}_{2}$ optimized with density functional theory (DFT). The frontier orbitals of free $\mathrm{MnPc}$ are doubly degenerate and correspond to the $e_{g}$ representation of the $\mathrm{D}_{4 h}$ point group, Figs. 4(b) and 4(d). Figure 4(c) shows a level scheme of a $d$-electron manifold in a tetrahedral ligand field for a neutral MnPc molecule. The $e_{g}$ orbitals correspond to the $d_{\pi}$ orbitals of the molecule. When the molecule captures an extra electron, degeneracy is lifted [Fig. 4(f)] and the resulting Jahn-Teller distortion leads to change in molecular orbital symmetry, Figs. 4(e) and 4(g). Simulated STM images, Figs. 4(h) and 4(i), correctly reproduce the experimental observation (see the Supplementary Material [29] for technical details).

The mean-field character of DFT leads to equal population of the two frontier orbitals [10]. However, a more realistic dynamical description would rather show the sequential population or depopulation of one orbital after the other, validating the DFT result as an average of dynamical configurations [3]. The experimental images in Figs. 2(c) and 2(f) can now be understood as corresponding to each of the two frontier orbitals if their degeneracy is lifted, where one is fully occupied and the other one is half filled. The full occupancy of the latter orbital is inhibited by the Coulomb interaction energy $e U_{C}$. Thus, the energy difference observed between the occupied and unoccupied molecular orbital in the STS signal $e U_{\text {sum }}$ [cf. black arrow in Fig. 2(g)] corresponds to the sum of $e U_{C}$ and the energy difference between the two orbitals $\Delta$.

Generally, degeneracy is primordial to Kondo effects, may it be a pure spin-related Kondo effect or the orbital Kondo effect, where two orbitals sequentially alter their population. The orbital Kondo effect is intrinsically related 


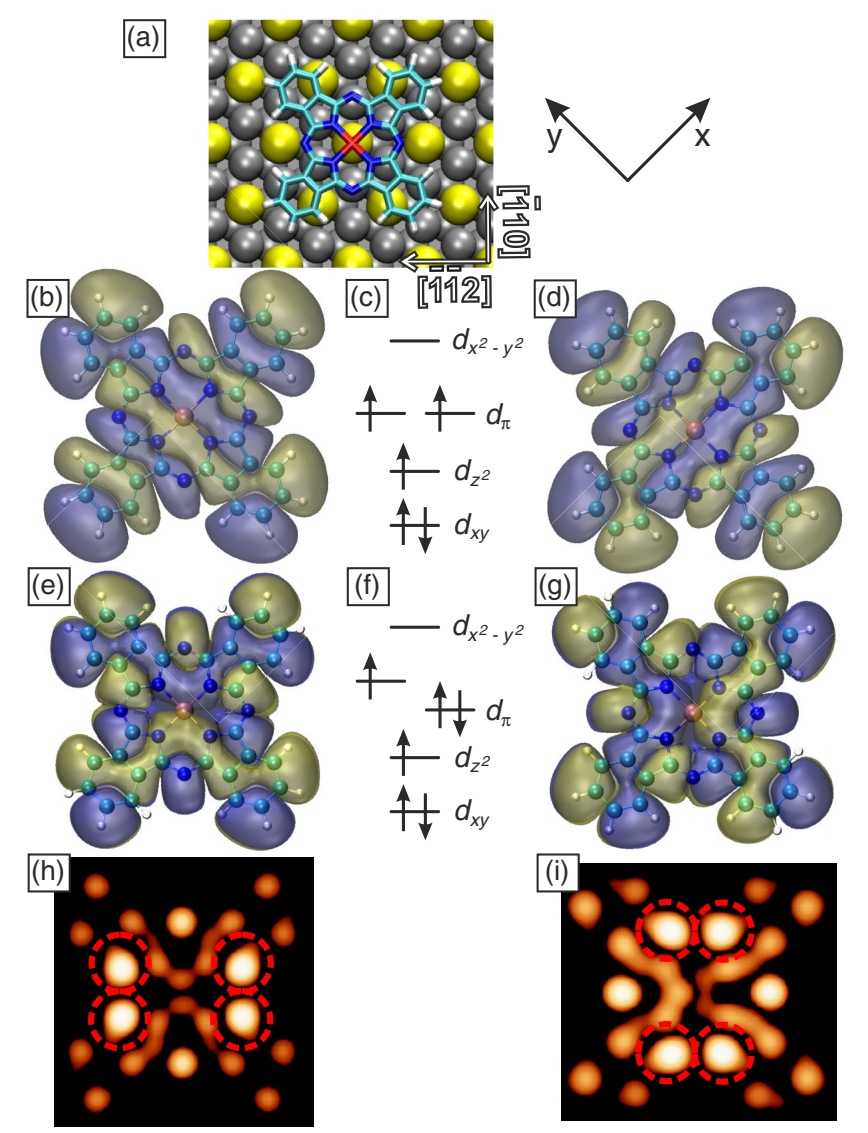

FIG. 4. (a) Adsorption geometry of $\mathrm{MnPc}$ on $\mathrm{SbAg}_{2}$ as obtained with DFT. The molecule is represented by a stick model (red, Mn; dark blue, N; light blue, C; white, H), the substrate by gray (Ag) and yellow spheres (Sb). (b),(d) Spatial map of the $d_{\pi}$ orbitals and (c) simplified level scheme showing the filling of Mn-related $d$ orbitals for the neutral molecule. (e), (g) $d_{\pi}$ orbitals of Jahn-Teller-distorted negatively charged MnPc. (f) Level spitting scheme of the charged molecule. (h), (i) Simulated STM image originating in the orbitals in (e) and (g), respectively. Red circles highlight the main orbital features as seen by STM.

to the SU(4) Kondo effect reported for carbon nanotubes, molecular systems, and quantum dots [7,42-44]. For the spin Kondo effect, the application of an external magnetic field leads to a Zeeman energy-induced lifting of spin degeneracy. Whereas the resulting energy gap at sufficiently low fields can still be overcome by spin-flip processes, large fields comparable to the Kondo formation energy eventually lead to the complete suppression of the Kondo effect [43,45-49]. In analogy, the lifting of orbital degeneracy associated with the reduction from SU(4) to SU(2) symmetry $[46,47,50,51]$ leads to the disappearance of the orbital Kondo effect and inelastic steps associated with the occupation transfer between the two orbitals, the spatial distribution of which is schematically represented in Fig. 5(a). Specifically, in the case for MnPc on $(\sqrt{3} \times \sqrt{3})$ reconstructed surfaces, the Jahn-Teller effect accounts for the lifting of degeneracy of the $C_{2 v}$ orbitals with

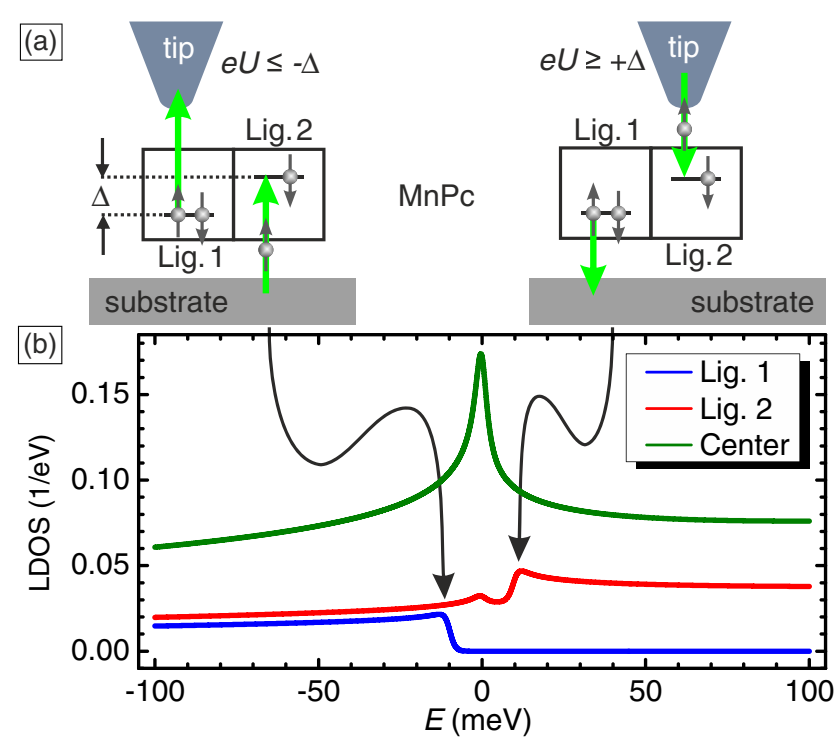

FIG. 5. (a) Scheme of the isospin-flip measurement process. In case of a negative (positive) bias polarity as presented in the left (right) panel for $e U \leq-\Delta(e U \geq \Delta)$, an electron from the substrate (tip) can fill the empty state of the Lig. 2 orbital accompanied by a electron transfer from the Lig. 1 orbital to the tip (substrate). (b) Projected density of states on the occupied (blue curve) and the empty orbital (red curve), both corresponding to the $d_{x z}$ and $d_{y z}$ manifold, and the $d_{z^{2}}$ one (green curve). The red orbital shows an $S=1 / 2$ Kondo feature with a low Kondo temperature.

contributions from $d_{x z}$ and $d_{y z}$ ion states. Its strength depends on the coupling of the molecule with the substrate. The experimental thresholds of $\mathrm{MnPc}$ on $\mathrm{BiCu}_{2}, \mathrm{BiAg}_{2}$, and $\mathrm{SbAg}_{2}$ are between 9 and $15 \mathrm{meV}$. These values are in good agreement with Jahn-Teller energies of MnPc in the gas phase ranging from 17 to $22 \mathrm{meV}$ [10]. A down-shift of these values is expected due to the adsorption-induced softening of the molecular modes at the origin of the Jahn-Teller effect.

Using the experimental inelastic threshold, we simulate the dynamical electronic structure via an Anderson Hamiltonian using two orbitals with identical one-electron coupling to the metal substrate. We solve the electronic structure using the multiorbital noncrossing approximation [30,31,52], which takes into account the charge fluctuation between the different charge states of the molecule rendered possible by hybridization with the substrate, and obtain the spectral function or density of states projected (PDOS) onto each orbital. Figure 5(b) shows the PDOS on each of the $C_{2 v}$ orbitals. The low-energy orbital shows a step at negative bias, whereas the high-energy one displays the step at positive energy. This is in agreement with our $d I / d U$ measurements and the symmetry of the ground and excited states [31]. Since the ground state of the molecule corresponds to populating the low-energy orbital, the PDOS reflects that a hole injected in the ground state leads to the excitation. Conversely, the excited state corresponds to injecting one electron into the high-energy 
orbital; thus the polarity of the conductance step is associated with the PDOS of each orbital, in excellent agreement with the experimental findings. When the tip is located at the center of the molecule, the density of states is maximized for the $d_{z^{2}}$ orbital of the Mn atom. This orbital is partially filled when the molecule is in contact with the substrate and closer to the Fermi energy than the $d_{x z}$ and $d_{y z}$ orbitals. The consequence is that the corresponding Kondo temperature is noticeably larger and a Kondo peak is visible at the experimental temperature.

In summary, the Jahn-Teller-split frontier orbitals of a single $\mathrm{MnPc}$ molecule adsorbed on different metallic substrates have been revealed by measuring the local tunneling differential conductances. A pronounced inelastic threshold appears at negative bias for the low-energy orbital and at positive bias for the high-energy one. This is the hallmark of an isospin-flip excitation as revealed by many-body calculations of the dynamical occupation of the two orbitals. Correlation effects impede the simultaneous population of both orbitals but result in fluctuations. In the absence of Jahn-Teller splitting, these fluctuations would lead to the well-known orbital Kondo effect.

N. L. thanks Roberto Robles for his data on the $\mathrm{SbAg}_{2}$ substrate and on the MnPc molecule. Financial support has been granted by MINECO (MAT2015-66888-C3-2-R and MAT2016-78293-C6-6-R) and FEDER funds. M. Bode acknowledges financial support by DFG through SFB 1170 "ToCoTronics" (project C02).

*jens.kuegel@physik.uni-wuerzburg.de

†Present address: Department of Physics, National Tsing Hua University, 30013 Hsinchu, Taiwan.

*Present address: CNRS, CEMES (Centre d'Elaboration de Matériaux et d'Etudes Structurales), B.P. 94347, 29 Rue Jeanne Marvig, 31055 Toulouse, France.

${ }^{\S}$ Present address: Max-Planck-Institut für Festkörperforschung, Heisenbergstraße 1, 70569 Stuttgart, Germany.

"Present address: Instituto de Ciencia de Materiales de Aragón, CSIC-Universidad de Zaragoza, Pedro Cerbuna 12, 50009, Spain.

[1] M. Ternes, A. J. Heinrich, and W.-D. Schneider, J. Phys. Condens. Matter 21, 053001 (2009).

[2] D. K. Morr, Rep. Prog. Phys. 80, 014502 (2017).

[3] D.-J. Choi and N. Lorente, in Handbook of Materials Modeling: Applications: Current and Emerging Materials, edited by W. Andreoni and S. Yip (Springer International Publishing, Cham, 2018), pp. 1-32.

[4] K. von Bergmann, M. Ternes, S. Loth, C. P. Lutz, and A. J. Heinrich, Phys. Rev. Lett. 114, 076601 (2015).

[5] D. L. Cox and A. Zawadowski, Adv. Phys. 47, 599 (1998).

[6] O. Y. Kolesnychenko, R. de Kort, M. I. Katsnelson, A. I. Lichtenstein, and H. van Kempen, Nature (London) 415, 507 (2002).

[7] P. Jarillo-Herrero, J. Kong, H. S. J. van der Zant, C. Dekker, L. P. Kouwenhoven, and S. D. Franceschi, Nature (London) 434, 484 (2005).
[8] M. Karolak, D. Jacob, and A. I. Lichtenstein, Phys. Rev. Lett. 107, 146604 (2011).

[9] C. Uhlmann, I. Swart, and J. Repp, Nano Lett. 13, 777 (2013).

[10] L. Andjelkovic, S. Stepanovic, F. Vlahovic, M. Zlatar, and M. Gruden, Phys. Chem. Chem. Phys. 18, 29122 (2016).

[11] Isospin is used here in the sense defined by $\mathrm{Li}$ et al. [22], where spin degeneracy and a twofold orbital degeneracy (isospin) are considered.

[12] B. C. Stipe, M. A. Rezaei, and W. Ho, Science 280, 1732 (1998).

[13] T. Komeda, Prog. Surf. Sci. 78, 41 (2005).

[14] K. Morgenstern, N. Lorente, and K.-H. Rieder, Phys. Status Solidi (b) 250, 1671 (2013).

[15] A. J. Heinrich, J. A. Gupta, C. P. Lutz, and D. M. Eigler, Science 306, 466 (2004).

[16] C. Krull, R. Robles, A. Mugarza, and P. Gambardella, Nat. Mater. 12, 337 (2013).

[17] J. Brede, N. Atodiresei, S. Kuck, P. Lazić, V. Caciuc, Y. Morikawa, G. Hoffmann, S. Blügel, and R. Wiesendanger, Phys. Rev. Lett. 105, 047204 (2010).

[18] R. Robles, N. Lorente, H. Isshiki, J. Liu, K. Katoh, B. K. Breedlove, M. Yamashita, and T. Komeda, Nano Lett. 12, 3609 (2012).

[19] Y.-H. Zhang, S. Kahle, T. Herden, C. Stroh, M. Mayor, U. Schlickum, M. Ternes, P. Wahl, and K. Kern, Nat. Commun. 4, 2110 (2013).

[20] M. Ormaza, P. Abufager, B. Verlhac, N. Bachellier, M.-L. Bocquet, N. Lorente, and L. Limot, Nat. Commun. 8, 1974 (2017).

[21] S. Karan, C. Garcia, M. Karolak, D. Jacob, N. Lorente, and R. Berndt, Nano Lett. 18, 88 (2018).

[22] Y. Q. Li, M. Ma, D. N. Shi, and F. C. Zhang, Phys. Rev. Lett. 81, 3527 (1998).

[23] L. El-Kareh, P. Sessi, T. Bathon, and M. Bode, Phys. Rev. Lett. 110, 176803 (2013).

[24] L. El-Kareh, G. Bihlmayer, A. Buchter, H. Bentmann, S. Blügel, F. Reinert, and M. Bode, New J. Phys. 16, 045017 (2014).

[25] L. Moreschini, A. Bendounan, H. Bentmann, M. Assig, K. Kern, F. Reinert, J. Henk, C. R. Ast, and M. Grioni, Phys. Rev. B 80, 035438 (2009).

[26] M. Steinbrecher, H. Harutyunyan, C. R. Ast, and D. Wegner, Phys. Rev. B 87, 245436 (2013).

[27] D. Jacob, M. Soriano, and J. J. Palacios, Phys. Rev. B 88, 134417 (2013).

[28] E. Minamitani, Y.-S. Fu, Q.-K. Xue, Y. Kim, and S. Watanabe, Phys. Rev. B 92, 075144 (2015).

[29] See Supplemental Material at http://link.aps.org/ supplemental/10.1103/PhysRevLett.121.226402 for an analysis of $\mathrm{MnPc} / \mathrm{BiAg}_{2}$ and details on the methods of theory calculations, which includes Refs. [3,27,30-40].

[30] J. Kroha, S. Kirchner, G. Sellier, P. Wölfle, D. Ehm, F. Reinert, S. Hüfner, and C. Geibel, Physica (Amsterdam) 18E, 69 (2003).

[31] R. Korytár and N. Lorente, J. Phys. Condens. Matter 23, 355009 (2011).

[32] G. Kresse and J. Hafner, Phys. Rev. B 47, 558 (1993).

[33] G. Kresse and J. Hafner, Phys. Rev. B 48, 13115 (1993).

[34] G. Kresse and J. Furthmüller, Comput. Mater. Sci. 6, 15 (1996). 
[35] G. Kresse and J. Furthmüller, Phys. Rev. B 54, 11169 (1996).

[36] G. Kresse and D. Joubert, Phys. Rev. B 59, 1758 (1999).

[37] S. Grimme, J. Antony, S. Ehrlich, and H. Krieg, J. Chem. Phys. 132, 154104 (2010).

[38] J. Tersoff and D. R. Hamann, Phys. Rev. B 31, 805 (1985).

[39] M.-L. Bocquet, H. Lesnard, S. Monturet, and N. Lorente, Computational Methods in Catalysis and Materials Science (Wiley-Blackwell, Weinheim, Germany, 2009), pp. 199-219.

[40] A. Mugarza, R. Robles, C. Krull, R. Korytár, N. Lorente, and P. Gambardella, Phys. Rev. B 85, 155437 (2012).

[41] L. Moreschini, A. Bendounan, H. Bentmann, M. Assig, K. Kern, F. Reinert, J. Henk, C. R. Ast, and M. Grioni, Phys. Rev. B 80, 035438 (2009).

[42] E. Minamitani, N. Tsukahara, D. Matsunaka, Y. Kim, N. Takagi, and M. Kawai, Phys. Rev. Lett. 109, 086602 (2012).

[43] A. W. Holleitner, A. Chudnovskiy, D. Pfannkuche, K. Eberl, and R. H. Blick, Phys. Rev. B 70, 075204 (2004).
[44] A. J. Keller, S. Amasha, I. Weymann, C. P. Moca, I. G. Rau, J. A. Katine, H. Shtrikman, G. Zaránd, and D. GoldhaberGordon, Nat. Phys. 10, 145 (2014).

[45] J. Paaske, A. Rosch, P. Wölfle, N. Mason, C. M. Marcus, and J. Nygard, Nat. Phys. 2, 460 (2006).

[46] M.-S. Choi, R. López, and R. Aguado, Phys. Rev. Lett. 95, 067204 (2005).

[47] J. S. Lim, M.-S. Choi, M. Y. Choi, R. López, and R. Aguado, Phys. Rev. B 74, 205119 (2006).

[48] R. Korytár, N. Lorente, and J.-P. Gauyacq, Phys. Rev. B 85, 125434 (2012).

[49] D. Jacob and J. Fernández-Rossier, Eur. Phys. J. B 89, 210 (2016).

[50] L. Tosi, P. Roura-Bas, and A. Aligia, Physica (Amsterdam) 407B, 3259 (2012).

[51] V. Lopes, R. A. Padilla, G. B. Martins, and E. V. Anda, Phys. Rev. B 95, 245133 (2017).

[52] Y. Kuramoto, Z. Phys. B 53, 37 (1983). 